Br. J. Surg. 1993, Vol. 80, December, 1575-1578

J. H. G. Klinkenbijl,

J. Jeekel,

P. I. M. Schmitz*,

P. A. R. Rombout $\dagger$,

G. A. J. J. Nix†,

H. A. Bruining and

$M$. van Blankenstein $\ddagger$

Departments of General Surgery and †Radiology, University

Hospital Dijkzigt, *Dr Daniel den Hoed Clinic and Department of

Biostatistics, Erasmus University and $\$$ Department of Internal

Medicine II, Erasmus University

Hospital, Rotterdam,

The Netherlands

Correspondence to:

Professor J. Jeekel, Department of

General Surgery, Erasmus

University Hospital,

Dr Molewaterplein 40, 3015-GD

Rotterdam, The Netherlands

\section{Carcinoma of the pancreas and periampullary region: palliation versus cure}

\begin{abstract}
A retrospective study of 310 patients with carcinoma of the head of the pancreas or periampullary region was performed. Preoperative bile drainage by placement of a stent reduced the number of postoperative complications, especially bleeding $(\mathrm{P}=0 \cdot 03)$. The operative mortality rate was nil in patients with periampullary cancer aged under 70 years and 23 per cent in those over 70 years of age $(\mathrm{P}<0.001)$. In the last 2 years of the study, the mortality rate following resection decreased to 2 per cent. Tumour-containing resection margins did not influence survival after resection $(\mathrm{P}=0.48)$. Tumour dimension of pancreatic and periampullary cancer and the presence of tumour in locoregional lymph nodes $\left(N_{l a}\right)$ resected with the primary tumour in cancer of the head of the pancreas were of no prognostic value. Following palliative resection of carcinoma of the pancreatic head, median survival was significantly better than when no resection was performed (10.1 versus 3.9 months, $\mathrm{P}<0.001$ ). In conclusion, even palliative resection may benefit some patients. Preoperative bile drainage is indicated in those with jaundice. Resection should be performed, irrespective of tumour size, provided that the unit's operative mortality rate is sufficiently low.
\end{abstract}

In patients with cancer of the head of the pancreas, pancreatoduodenectomy (Whipple's procedure) is the treatment of choice in most centres. However, the tumour proves to be irresectable in many cases and 90 per cent of all patients admitted to hospital with pancreatic cancer die within 1 year. The only chance of prolonged survival lies in radical tumour resection. After surgical treatment with curative intent, a 5-year survival rate of only $0-15$ per cent has been found and about 80 per cent of patients die within 2 years of resection. Efforts ${ }^{1-4}$ to improve survival by extending the resection to total pancreatectomy or regional extended pancreatectomy as described by Fortner ${ }^{5}$ have not met with great success.

The question therefore arises as to whether surgical resection is the best treatment for pancreatic cancer. The operative mortality and morbidity rates are high, and operative risks should be weighed against any expected gain in survival. The operative mortality rate over the years 1981-1986 reported in a recent review of 2398 pancreatic resections from different centres was 16 per cent. Nevertheless, improvement has been made in recent years and even a 0 per cent mortality rate has been reported ${ }^{6,7}$.

Operation for cancer of the pancreas can be potentially radical if the malignant process is still confined to the resected area. Before operation an attempt should be made to select patients who have a limited tumour mass without distant metastases or lymph node involvement outside the resection area. At present, no techniques are available to identify such patients accurately. In a previous study ${ }^{8,9}$, one general risk factor for operative mortality was identified: patients aged over 70 years had an operative mortality rate of 27 per cent compared with 3 per cent in those less than 70 years of age.

In this study, the results of treatment in 310 patients admitted in the period 1977-1988 were analysed retrospectively to determine the influence of preoperative symptoms, preoperative biliary drainage, tumour size, tumour-containing resection margins and lymph node involvement on the outcome of surgical resection in cancer of the head of the pancreas and periampullary region.

\section{Patients and methods}

Between 1977 and 1988, 310 patients were admitted to the Department of Surgery of the University Hospital Dijkzigt, Rotterdam, for cancer of the head of the pancreas or periampullary region. Standard preoperative investigation included ultrasonography, computed tomography (CT), endoscopic retrograde cholangiopancreatography (ERCP) or percutaneous transhepatic cholangiography (PTC) and optional angiography of the coeliac and superior mesenteric arteries.

The mean age of the patients was 62.6 (range 26-89) years. There were 180 men and 130 women. Cancer of the head of the pancreas was diagnosed in 226 patients. No operation was performed in 59 of these patients ( 26 per cent) because of distant metastases or a bad World Health Organization performance rating. Clinical history, preoperative diagnostic procedures, intraoperative and pathological findings, and follow-up data were analysed using the log rank test, the Cox regression model and Kaplan-Meier life-tables.

The standard operation for resectable cancer was Whipple's procedure. A jejunal loop was used for reconstruction, with end-to-side or end-to-end pancreatojejunostomy with a one-layer inverting running suture. An end-to-side choledochojejunostomy was constructed on the same jejunal loop, also with a one-layer running suture. Stents were not used for the pancreatic duct and in only a few cases for the bile duct. In the case of positive frozen-section examination of the pancreatic resection margin, subtotal or total pancreatectomy was carried out. Pancreatic resection was performed by a limited number of surgeons. Operative mortality was defined as death within 30 days of operation or during the first admission to hospital. Tumour node metastasis (TNM) staging after operation was as defined in the Union Internacional Contra la Cancrum 1987 system, with a modification for the $\mathbf{N}$ stage, stage $\mathbf{N}_{13}$ indicating positive lymph nodes within the resection specimen and $N_{1 b}$ positive nodes outside the specimen.

\section{Results}

Of a total of 242 operations for carcinoma of the head of the pancreas $(n=167)$ or periampullary region $(n=75)$, a radical resection with curative intent could be performed in 106 patients (34 per cent): 50 with cancer of the head of the pancreas ( 30 per cent resection rate) and 56 with periampullary cancer ( 75 per cent resection rate). In 19 patients (6 per cent) a 
palliative, i.e. non-radical, resection was performed. Nonradicality was shown after operation by pathological examination: stage $T_{3}$ pancreatic cancer (seven patients) and $\mathrm{T}_{4}$ periampullary cancer (three), $\mathrm{N}_{1 \mathrm{~b}}$ (eight) and $\mathrm{M}_{1}$ (one). Stage $T_{4}$ periampullary carcinoma resembles stage $T_{3}$ pancreatic cancer, with tumour extending directly to any of the stomach, spleen, colon or adjacent large vessels. In 34 patients with infiltration of tumour into vital organs or distant metastasis to lymph nodes or liver, the operation was limited to an exploratory laparotomy. To treat or prevent biliary or gastric outlet obstruction, a drainage procedure, i.e. choledochojejunostomy and/or gastrojejunostomy, was performed in 83 patients.

Preoperative stent drainage was established by means of PTC or ERCP in 88 patients ( 39 per cent) with cancer of the head of the pancreas. In 16 patients (7 per cent) stenting was undertaken during operation as a palliative procedure when none had been placed before surgery and no resection was possible. In patients with cancer of the periampullary region, a stent was placed before operation in 28 ( 33 per cent) and during operation in six ( 7 per cent). In total, 138 patients (45 per cent) received an endoprosthesis. In 38 patients no treatment other than stenting was possible.

In 40 per cent of all patients ( 125 of 310 ) malignancy was cytologically or histologically confirmed before operation.

\section{Patient details}

Cancer of the head of the pancreas. Presenting symptoms in 226 patients with carcinoma of the pancreatic head are listed in Table 1. Pain, weight loss, jaundice and increased levels of alkaline phosphatase and $\gamma$-glutamyl transferase were the main signs. Of patients with pain, distant metastases were found in 97 (62 per cent) during preoperative investigation and, as a

Table 1 Preoperative clinical features in patients with cancer of the head of the pancreas or periampullary region

\begin{tabular}{lll}
\hline & $\begin{array}{l}\text { Head of the } \\
\text { pancreas } \\
(n=226)\end{array}$ & $\begin{array}{l}\text { Periampullary } \\
\text { region } \\
(n=84)\end{array}$ \\
\hline Pain & $157(69)$ & $40(48)$ \\
Weight loss & $173(77)$ & $57(68)$ \\
Gastric stasis & $67(30)$ & $16(19)$ \\
Jaundice & $160(71)$ & $61(73)$ \\
Altered defaecation & $72(32)$ & $22(26)$ \\
Raised alkaline phosphatase & $142(63)$ & $68(81)$ \\
Raised $\gamma$-glutamyl transferase & $168(74)$ & $56(67)$ \\
Raised lactic dehydrogenase & $78(35)$ & $22(26)$ \\
Coagulation disorder & $38(17)$ & $24(29)$ \\
\hline
\end{tabular}

Values in parentheses are percentages

Table 2 Surgical procedures performed

\begin{tabular}{|c|c|c|c|}
\hline & $\begin{array}{l}\text { Head of the } \\
\text { pancreas } \\
(n=226)\end{array}$ & $\begin{array}{l}\text { Periampullary } \\
\text { region } \\
(n=84)\end{array}$ & $\begin{array}{l}\text { Total } \\
(n=310)\end{array}$ \\
\hline No operation & $59(26)$ & $9(11)$ & $68(22)$ \\
\hline $\begin{array}{l}\text { Exploratory } \\
\text { laparotomy }\end{array}$ & $30(13)$ & $4(5)$ & $34(11)$ \\
\hline Drainage procedure & $74(33)$ & $9(11)$ & $83(27)$ \\
\hline Palliative resection & $13(6)$ & $6(7)$ & $19(6)$ \\
\hline \multicolumn{4}{|l|}{ Curative resection } \\
\hline Whipple's procedure & $30(13)$ & $41(49)$ & $71(23)$ \\
\hline PPPD & $8(4)$ & $14(17)$ & $22(7)$ \\
\hline Total pancreatectomy & $12(5)$ & $1(1)$ & $13(4)$ \\
\hline Total & $50(22)$ & $56(67)$ & $106(34)$ \\
\hline $\begin{array}{l}\text { Tumour-containing } \\
\text { resection margin }\end{array}$ & 9 of $50(18)$ & 4 of $56(7)$ & 13 of $106(12)$ \\
\hline
\end{tabular}

Values in parentheses are percentages. PPPD, pylorus-preserving pancreatoduodenectomy
Table 3 Hospital mortality after operation for pancreatic and periampullary cancer

\begin{tabular}{|c|c|c|c|c|}
\hline \multirow[b]{3}{*}{ Site of tumour } & \multicolumn{3}{|c|}{ No. of deaths } & \multirow[b]{3}{*}{$P^{*}$} \\
\hline & \multirow[b]{2}{*}{$\begin{array}{l}\text { Total } \\
(n=242)\end{array}$} & \multicolumn{2}{|c|}{ Age (years) } & \\
\hline & & $\begin{array}{l}<70 \\
(n=164)\end{array}$ & $\begin{array}{l}\geqslant 70 \\
(n=78)\end{array}$ & \\
\hline \multicolumn{5}{|l|}{ Head of the pancreas } \\
\hline Overall $(n=167)$ & $21(13)$ & $9(7)$ & $12(27)$ & $<0.05$ \\
\hline After resection $(n=50)$ & $4(8)$ & $3(7)$ & $1(14)$ & n.s. \\
\hline \multicolumn{5}{|l|}{ Periampullary region } \\
\hline Overall $(n=75)$ & $5(7)$ & $1(2)$ & $4(17)$ & $<0.001$ \\
\hline After resection $(n=56)$ & $3(5)$ & $0(0)$ & $3(23)$ & $<0.001$ \\
\hline Total $(n=242)$ & $26(11)$ & $10(6)$ & $16(21)$ & n.s. \\
\hline
\end{tabular}

Values in parentheses are percentages. $*<70$ versus $\geqslant 70$ years (log rank test); n.s., not significant

consequence, no operation or surgical drainage procedure was performed. Coagulation disorders were all the result of vitamin $\mathrm{K}$ deficiency. In 54 patients ( 24 per cent), tumour size was estimated before surgery by ultrasonography, CT and ERCP; in 15 the tumour was less than $3 \mathrm{~cm}$ in diameter.

The surgical procedures performed are shown in Table 2. Tumour in the resection margin was found in 20 patients with cancer of the head of the pancreas (11 after palliative Whipple's resection, nine after curative resection).

Cancer of the periampullary region. Eighty-four patients were admitted for carcinoma of the periampullary region, defined as a tumour in the ampulla of Vater ( 59 patients), distal common bile duct (CBD) (13) or duodenum (12). Weight loss, jaundice and raised levels of alkaline phosphatase and $\gamma$-glutamyl transferase were the main presenting features in this group (Table 1). Surgical procedures are listed in Table 2. Six patients with periampullary cancer had tumour growth in the resection margin (two after palliative, four after curative resection ).

\section{Postoperative complications}

Cancer of the head of the pancreas. After resection, 14 re-explorations ( 22 per cent of patients) were needed, ten for intra-abdominal bleeding, one for leakage of the pancreatojejunostomy and three for various reasons. Two wound abscesses were drained on the ward and one intra-abdominal abscess treated conservatively, as were 12 other minor complications. Non-surgical complications (cardiac, respiratory and urinary tract) occurred in 45 patients $(27$ per cent).

Cancer of the periampullary region. In this group, 14 re-explorations ( 19 per cent of patients) were carried out, four for intra-abdominal bleeding, two for intra-abdominal abscess, three for leakage of the pancreatojejunostomy and five for other reasons. In one patient, leakage of the pancreatojejunostomy was treated conservatively, as were eight other minor complications. Non-surgical complications occurred in 23 patients ( 31 per cent).

Preoperative drainage. No preoperative drainage for jaundice was performed in 170 patients; 116 had an endoprosthesis inserted for preoperative biliary drainage. Postoperative laparotomy for bleeding occurred in two patients who underwent stenting before operation and in 14 with no stent $\left(\chi^{2}=4 \cdot 60,1\right.$ d.f., $\left.P=0.03\right)$, probably due to disturbance of coagulation as a result of impaired liver function.

\section{Operative mortality}

The hospital mortality rate after all surgical interventions was 11 per cent. Patients aged over 70 years had a higher operative risk (Table 3). However, this was significant only in overall 
mortality after all surgical interventions for patients with cancer of the pancreatic head; following resection the difference was not significant. During the last 2 years of study the operative mortality rate after curative resection has fallen to 2 per cent (one patient) in the last 40 procedures.

\section{Pathology}

Histological investigation of resection specimen margins revealed a high proportion containing tumour, 18 per cent in cancer of the head of the pancreas and 7 per cent in that of the periampullary region. A tumour-containing resection margin was found in five of ten pylorus-preserving pancreatic resections, but no duodenal resection margins containing tumour were found. Almost all tumours were moderately to poorly differentiated adenocarcinoma. In 56 of 78 patients with liver metastases and in 11 of 22 with peritoneal carcinomatosis, histological confirmation of the metastases was obtained

\section{Survival and prognostic factors}

Cancer of the head of the pancreas. Overall survival in this group of patients was 23 per cent at 1 year and 3 per cent at 5 years, with a median survival of 5.1 months. There were significant differences in survival depending on treatment. When no resection was performed, the 1-year survival rate was significantly lower than that in patients undergoing palliative resection (11 versus 38 per cent respectively). The longest survival was achieved after intentional curative resection, with survival rates of 56,28 and 11 per cent at 1,2 and 5 years respectively (median 13.3 months) (Figure 1).

Involvement of local lymph nodes $\left(\mathrm{N}_{1 \mathrm{a}}\right)$ did not influence survival after resection with curative intent. In 36 patients with stage $\mathrm{N}_{0}$ and 14 with stage $\mathrm{N}_{1 \mathrm{a}}$ cancer, a 1-year survival rate of 61 and 35 per cent and a 2 -year survival rate of 28 and 20 per cent respectively were observed $(P=0.43)$. Higher age $(P=0.01)$ and pain $(P=0.003)$ were of prognostic value for overall survival in pancreatic cancer, as well as a $\gamma$-glutamyl transferase level above 250 units $/ 1(P<0.02)$ and a lactic dehydrogenase concentration greater than 320 units/1 $(P<0.002)$. Tumour dimension measured in the resection specimen directly after operation did not have significant prognostic value. In contrast, tumour size measured before operation showed an unexpected trend $(P=0.08)$ : patients with larger tumours had a trend towards improved survival. A positive resection margin was found in 11 patients after palliative and in nine after curative resection. Following resection with curative intent, survival was not influenced by microscopic tumour growth in the resection margin $(P=0 \cdot 48)$. After multivariate analysis of the prognostic factors significant on univariate analysis, age above 70 years, pain and a lactic

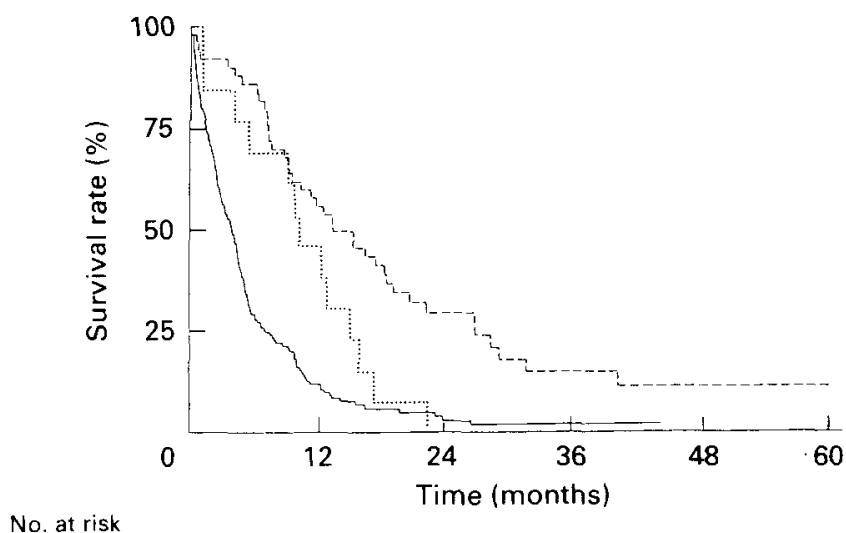

$\begin{array}{lrcclll}\text { Curative } & 50 & 28 & 14 & 7 & 6 & 6 \\ \text { Palliative } & 13 & 5 & 0 & 0 & 0 & 0 \\ \text { No resection } & 163 & 18 & 3 & 0 & 0 & 0\end{array}$

Figure 1 Survival related to treatment in patients with cancer of the head of the pancreas. - , No resection; ---, curative resection; $\cdots$. palliative resection. $\mathrm{P}<0.001$ ( log rank test) dehydrogenase level over 320 units/1 remained of significant value for prognosis as independent factors.

Cancer of the periampullary region. The overall survival rate at 1 year was 65 per cent, at 2 years 45 per cent and at 5 years 28 per cent. Significant improvements in survival were found in this group of patients after both palliative and curative resection compared with no resection $(P<0.001)$. After resection with curative intent, survival rates of 78,64 and 43 per cent were obtained at 1,2 and 5 years respectively (median 54.8 months).

There was no difference in survival between 40 patients with stage $\mathrm{N}_{0}$ and 16 with stage $\mathrm{N}_{1 \mathrm{a}}$ cancer, with a 1-year survival rate of 82 and 65 per cent, a 2-year rate of 70 and 50 per cent, and a 5 -year rate of 43 and 42 per cent respectively $(P=0.43)$. However, comparison of survival in these two groups of patients with Greenwood confidence intervals suggests that a significant difference would be found if more patients were included. Age $(P=0.01)$ was a significant prognostic factor for overall survival. Pain $(P=0.83)$ and raised $\gamma$-glutamyl transferase $(P=0.77)$ and lactic dehydrogenase $(P=0.31)$ levels had no influence on survival. Tumour-containing resection margins $(P=0.23)$ and tumour size $(P=0.24)$ had no prognostic value. Multivariate analysis showed that only age above 70 years was of prognostic value as an independent factor.

\section{Discussion}

Only a small proportion of patients with pancreatic cancer will survive more than 3 years after resection. Apparently the tumour process has metastasized beyond the resection area in most patients at the time of surgery, and this cannot be recognized adequately before or during operation. Better selection criteria are therefore needed to achieve successful curative surgery. In this retrospective study, an attempt has been made to identify variables that might predict outcome or help to select appropriate patients for surgery. The authors accept the limitations of the method, but prospective controlled studies are currently lacking.

The chosen definition of operative mortality is reliable as it includes all deaths related to operation, not only those occurring in the 30-day period. The operative mortality rate after resection was 8 per cent in patients with cancer of the head of the pancreas. The lack of any difference between those aged more or less than 70 years probably reflects careful selection for operation in the older patients. In periampullary cancer in patients aged under 70 years, there were no postoperative deaths, which is significantly different from the mortality rate of 23 per cent found in patients over 70 years of age $(P<0.001)^{8,9}$. The operative mortality rate decreased progressively in the last 2 years of the study to 2 per cent in the most recent 40 resections. A similar low operative mortality rate has been reported by others?

Surgical drainage procedures for irresectable tumours carry a high operative mortality rate (about 10 per cent) and should therefore be performed only in the presence of gastric or duodenal obstruction when endoscopic stenting for jaundice is impossible. In this situation, when an operation has to be performed, the addition of gastroenterostomy is advised by some authors ${ }^{10-13}$. The present authors' opinion is that bilioenteric anastomosis should not be combined with gastroenterostomy if the patient has no duodenal obstruction ${ }^{14}$.

Preoperative relief of jaundice by stenting the CBD by means of ERCP or PTC resulted in a significant reduction in the number of postoperative laparotomies for bleeding and fewer cases of abscess and anastomotic leakage. Jaundice should be treated as early as possible before operation ${ }^{9,15}$. Resection should be performed only in the non-jaundiced patient.

Non-radical resection was demonstrated in 19 patients by pathological examination, which showed tumour cells in regional lymph nodes outside the resection specimen or even distant metastases. Despite this non-radicality, the prognosis of these patients after palliative resection was significantly better 
than that in those who underwent surgical drainage or exploratory laparotomy. Resection margins that appeared to contain tumour in the definitive pathological specimen did not appear to influence survival in the present study. Attempts to avoid non-radical resection by extending the area of excision have not been very promising so far ${ }^{5}$. Recently, however, a study from Japan ${ }^{16}$ showed significantly reduced local recurrence rates after extended clearance of regional $\left(R_{2}\right)$ lymph nodes.

In the present series of 310 patients admitted for pancreatic or periampullary cancer, potentially curative resection could be performed in only 106 ( 34 per cent ). After curative resection for cancer of the head of the pancreas, 56 per cent of patients survived for more than 1 year, and 2- and 5-year survival rates were 28 and 11 per cent. These survival data are similar to those encountered in other studies ${ }^{17-26}$. In a recent review ${ }^{6}$ of 4100 pancreatic resections for cancer, a 5-year survival rate of 4 per cent was found.

In the present study, independent prognostic factors for overall survival were found for patients with cancer of the head of the pancreas. Age above 70 years, pain and a lactic dehydrogenase level greater than 320 units $/ 1$ correlated with a decreased survival rate. In periampullary cancer, only age above 70 years remained as an independent prognostic factor. In both forms of cancer, tumour size had no prognostic value. Thus, a larger tumour does not necessarily imply a worse prognosis; in the present study, a larger preoperative tumour diameter showed a trend towards a better patient survival rate $(P=0.08)$. Tumour infiltration of locoregional lymph nodes $\left(\mathrm{N}_{1 \mathrm{a}}\right)$ resected together with the primary tumour did not appear to be of prognostic value in patients with cancer of the head of the pancreas.

In conclusion, pancreatic resection should be performed if technically possible, irrespective of tumour size or infiltration of locoregional lymph nodes (in patients with cancer of the pancreatic head). Non-radical resection may benefit selected patients, especially during the first year after resection. Although the present study suggests that surgical resection for cancer of the head of the pancreas is largely a palliative procedure, curing only a small proportion of patients, it is a good operation in centres with low operative mortality rates. As postoperative morbidity and mortality are increased in jaundiced patients and in those aged over 70 years, preoperative drainage of bile by endoscopic stenting of the distal CBD should be carried out in patients with jaundice and great caution is advised in the elderly.

\section{Acknowledgements}

The authors thank Miss J. Bakker for outstanding support and help in analysing the data.

\section{References}

1. Longmire WP. Cancer of the pancreas: palliative operation, Whipple procedure, or total pancreatectomy? World J Surg 1984; 8: $872-6$.

2. Ihse I, Lilja P, Arnesjö B, Bengmark S. Total pancreatectomy for cancer. An appraisal of 65 cases. Ann Surg 1977; 186: 675-80.

3. van Heerden JA. Pancreatic resection for carcinoma of the pancreas: Whipple versus total pancreatectomy - an institutional perspective. World J Surg 1984; 8: 880-8.

4. Cameron JL, Christ DW, Sitzmann JV et al. Factors influencing survival after pancreaticoduodenectomy for pancreatic cancer. Am J Surg 1991; 161: 120-4.

5. Fortner JG. Regional pancreatectomy for cancer of the pancreas, ampulla, and other related sites. Ann Surg 1984; 199: 418-25.

6. Gudjonssen B. Cancer of the pancreas: 50 years of surgery. Cancer 1987; 60: 2284-303.

7. Trede M. Survival after pancreatoduodenectomy; 118 consecutive resections without an operative mortality, Ann Surg 1990; 211: $447-58$

8. Obertop H, Bruining HA, Eeftinck Schattenkerk M, Eggink WF, Jeekel J, van Houten $H$. Operative approach to cancer of the head of the pancreas and periampullary region. Br J Surg 1982; 69. $573-6$.

9. Snellen JP, Obertop H, Bruining HA et al. The influence of preoperative jaundice, biliary drainage and age on postoperative morbidity and mortality after pancreatoduodenectomy and total pancreatectomy. Neth J Surg 1985; 37: 83-6.

10. La Ferla G, Murray WR. Carcinoma of the head of the pancreas: bypass surgery in unresectable disease. $B r J$ Surg 1987; 74: 212-13.

11. Potts JR III, Broughan TA, Hermann RE. Palliative operations for pancreatic carcinoma. Am J Surg 1990; 159: 72-8.

12. Singh SM, Longmire WP, Reber HA. Surgical palliation for pancreatic cancer: the UCLA experience. Ann Surg 1990; 212: 132-9.

13. Watanapa $\mathbf{P}$, Williamson RCN. Surgical palliation for pancreatic cancer: developments during the past two decades. $\mathrm{Br} J$ Surg 1992; 79: 8-20.

14. Brooks DC, Osteen RT, Gray EB, Steele GD, Wilson RE. Evaluation of palliative procedures for pancreatic cancer. $A m J$ Surg 1981; 141: 430-3

15. Gundrig SR, Siodll WC, Knol JA. Efficacy of preoperative biliary tract decompression in patients with obstructive jaundice. Arch Surg 1984; 119: 703-8.

16. Ishikawa $\mathrm{O}$, Ohigashi $\mathrm{H}$, Sasaki $\mathrm{Y}$ et al. Practical usefulness of lymphatic and connective tissue clearance for carcinoma of the pancreas head. Ann Surg 1988; 208: 215-20.

17. Piorkowski RJ, Blievernicht SW, Lawrence W Jr et al. Pancreatic and periampullary carcinoma. Am J Surg 1982; 143: 189-93.

18. Trede $M$. Treatment of pancreatic carcinoma: the surgeon's dilemma. Br J Surg 1987; 74: 79-80.

19. Moossa AR. Current status of surgical treatment of pancreatic cancer. Current Surgery 1982; 39: 297-8.

20. Cooperman AM, Herter FP, Marboe CA, Helmreich ZV Perzin KH. Pancreatoduodenal resection and total pancreatectomy - an institutional review. Surgery 1981; 90: 707-12.

21. Appelqvist $\mathbf{P}$, Virén $\mathbf{M}$, Minkkinen J, Kajanti M, Kostiainen S, Risanen $P$. Operative finding, treatment, and prognosis of carcinoma of the pancreas: an analysis of 267 patients. $J$ Surg Oncol 1983; 23: 143-50.

22. Edis AJ, Kiernan PD, Taylor WF. Attempted curative resection of ductal carcinoma of the pancreas. Review of Mayo Clinic experience, 1951-1975. Mayo Clin Proc 1980; 55: 531-6.

23. Warren KW, Christophi Chr, Armendariz R, Basu S. Current trends in the diagnosis and treatment of carcinoma of the pancreas. Am J Surg $1983 ; 145: 813-18$.

24. Trede $M$. The surgical treatment of pancreatic carcinoma. Surgery 1985; 97: 28-35

25. Kairaluoma MI, Stahlberg M, Kiviniemi H, Haukipuro K. Results of pancreatoduodenectomy for carcinoma of the head of the pancreas. Hepatogastroenterology 1989; 36: 412-18.

26. Funovics JM, Karner J, Pratschner Th, Fritsch A. Current trends in the management of carcinoma of the pancreatic head. Hepatogastroenterology $1989 ; 36: 450-5$.

Paper accepted 1 March 1993 